\title{
Recombinant Antibodies and Vaccines for Viruses
}

\section{Iqbal RK*and Shabir S}

Institute of Molecular Biology and Biotechnology, Bahauddin Zakariya University, Multan-60800, Pakistan

\begin{abstract}
Immunity produced when variable, diverse, and joining exons recombine and form diverse B-cell receptors. Somatic rearrangement of immunoglobulin genes termed VDJ recombination. This activity is controlled by RAG1 and RAG2 proteins, binds to the signal sequences and start cleavage. Double stranded breaks are produced by ROS, Nuclear enzymes and ATM. RAG protein induces cleavage activity. Segments exchange by CSR. After cleavage activity shuffling of segments occurs. TDT cause gain or loss of nucleotide bases. AID and RAG begins the process of CSR that shuffle exons of constant region. The core of NHEJ have catalytic subunit (DNA-Pkcs). Ku-DNA complex is important for the attachment of nuclease, polymerase and ligase of NHEJ. RAG2 with histone H3K4Me3 start recombination activity. HMG1 and HMG2 promote synapsis and cleavage. RNA Helicase of the DExD $\backslash H$ induces conformational changes. ZnA have ligase activity. Ku involved in the attachment of NHEJ factor DExD/H box who induce conformational changes. NHEJ machinery has XRCC4, XLF, and PAXX who ligate DNA ends. Protein kinase B and phosphoinositide-3 kinase involved in RNA expression. TOR69-3A2 is antibodies that neutralized Western equine encephalitis virus. AMMO1 is the anti $\mathrm{gH} / \mathrm{gL}$ monoclonal antibody prevent to the Epstein bar virus infection. Antibodies also used for Ebola virus and Hepatitis. WT and HVR1 gpE1/gpE2 produce antibodies which target any type of cross-genotype neutralizing epitopes for HCV. GPE118, GPE325, GPE534 are targeted to different epitopes for Ebola virus.
\end{abstract}

Keywords: Recombinant antibodies; Antigen binding sites; RAG1 and RAG2 complex; Non- homologous end joining

\section{INTRODUCTION}

\section{VDJ recombination}

Antibodies are basic part of humoral immunity. Recombinant antibodies are the important class of biotherapeutics and approved for many diseases. When humoral immunity is in the process, the variable, diversity and joining exon recombine and give rise to B-cell receptors, which generates antigen recognition structures these structures with the constant domain elements (IgM, IgD, IgG, and IgA) of immunoglobulin [1]. Antibodies identify antigens with high specificity. $F_{c}$ region of antibodies bind to the antigen $[2,3]$. Light chain has two subfamilies lambda and kappa. Kappa family mostly related to the antibodies those are marketed [4]. FAB is the antigen binding fragment which consists of variable region of heavy chain and light chain (VH and VL) and constant region [5]. Complementary determining region of variable region have loops involve in the interaction to the antigen $[6,7]$.

\section{Double stranded breaks}

In mature B-cells, SPIC maintain BCR signaling which is ordered by activation of NK-KB1. SPIC is bring out by DSBs. Whole abatement of pre-BCR tocsins by RAG DSBs depend on the deactivation of both SKY and BLNK [8].

\section{VDJ REGION’S GENE SHUFFLING}

Somatic rearrangement occurs in the immunoglobulin genes termed VDJ recombination. RSS bind to the RAG protein is traced when synapsis start [9]. Variable, diversity and joining region's gene shuffle and this shuffling generates genes that encode for the adjustable regions of T-cell receptor proteins and immunoglobulin's [10]. This activity is controlled by recombination - activating gene. Its form RAG1 and RAG2 proteins. RAG1 complete structure contain many regions perform different functions [11]. RAG1 is functional only in dimeric form and this form is important for complex formation with RAG2 [12].

\section{Recombinant signal sequence}

Synapses of Cis RSSs done more rapidly because it is kinetically favoured then trans RSSs [13]. RAG1 and RAG2 complex is lymphoid particular protein complex require for VDJ recombination. During

Correspondence to: Iqbal RK, Institute of Molecular Biology and Biotechnology, Bahauddin Zakariya University, Multan-60800, Pakistan, Tel: +9261 9210071; E-mail: Khalid.iqbal@bzu.edu.pk

Received: January 10, 2019, Accepted: January 25, 2019, Published: February 10, 2019

Citation: Iqbal RK (2019) Recombinant Antibodies and Vaccines for Viruses. J Biomol Res Ther, 8: 169. doi: 10.35248/2167-7956.19.8.169

Copyright: $@ 2019$ Iqbal RK, et al. This is an open access article distributed under the term of the Creative Commons Attribution License, which permits unrestricted use, distribution, and reproduction in any medium, provided the original work is properly cited. 
G1 phase of the cell cycle VDJ recombination begins when RAG recombinase generates double stranded fragments of VDJ coding segments and RSS [14]. RSSs are conserved heptamer and nanomer have consensus sequence of CACAGTG and ACAAAAA. When binds to the one RSS the cleavage start $[11,15]$. RAG first nick at 5' of the haptamer at the joining point present in between heptamer and VDJ coding segments. This shows that a $3^{\prime} \mathrm{OH}$ group at the end of the coding sides and a 5' phosphate group join with heptamer tip [16]. RAG1 have recombination signal sequence RSS-binding domain, this is the zone that reacts with RAG2 which also interact for DNA disunion with an active site [17]. Heptamer CACAGTG, a spacer region consist of $120-30 \mathrm{bp}$ and a nanomer [18]. In VDJ recombination two gene segments influence with each other, one from these genes have signal sequence. This signal sequence have 12-bps spacer signal and other of which have 23-bps space signal $[7,15,19]$. When RAG1, HMGB1 and RSS complex creation is greater in the presence of $\mathrm{Mn}^{2+}$ instead of $\mathrm{Mg}^{2+}$. The 12/23 synaptic complex formation in the presence of $\mathrm{Mg}^{2+}$ is linked to the configurational changes in the RAG complex [20]. Stoichiometry of RAG 2 is different between SC1 and SC2 met have one or two RAG units [21,22]. When RAG1 and RAG2 are mixed together produces RSS complex known as SC1 and SC2 [23]. According to $12 / 23$ Rule Process RAG complex identification of 12RSS and 23RSS produce correct recombination of a $\mathrm{V}$ segment with $\mathrm{J}$ segment or $\mathrm{V}$ segment recombination to a $\mathrm{D}$ segment and $\mathrm{D}$ segment joining with $\mathrm{J}$ segment [24,25]. VDJ recombination exploits DNA damage response to promote immunity $[9,25]$. The variety of DSBs produced due to abundantly different chemistry of DNA ends [26]. Double stranded breaks produced by metaphase chromosome and chromatic breaks during replication of nick, reactive oxygen species (ROS) and mitochondrion (SOD2) and cytosol (SOD1) also produce DSBs. Nuclear enzyme produce DSBs when topoisomerase not function [27]. AgR gene is important for adaptive immunity. AgR which are self-reactive cause apoptosis in immature lymphocyte. Ataxia telangiectasia mutated (ATM) kinase is responsible for the eukaryotic DSB response [28]. RAG cleavage of AgR loci that stimulate many signalling pathways and these pathways change the level of transcript [29].

\section{NON-HOMOLOGOUS END JOINING}

After cleavage activity by RAG protein, then joining of 3'OH group to the complimentary DNA strand performed by the process of trans-esterification. Terminal Deoxynucleotidyltransferase TDT, causes gain or loss of nucleotide bases [25]. This phenomena enhances survival of cell while double stranded breaks occurs [30]. The RAG proteins attaches to the signal end, cell withstands by the phosphorylation of GSK3B on Ser389 with the help of MAPK (mitogen activated protein kinase) [31]. DNA dependent protein kinase indicates the binary stranded breaks in cell cycle. HMGB (High Mobility Gene Binding Protein) have many proteins. Classical non-homologous end joining (C-NHEJ) is a major binary strand break's repair pathway by IgH class switched-NHEJ is made activated by class switch recombination in synaptic complex after cleavage CEs and SEs grasp by RAG complex [32]. Activation induced cytidine deaminase(AID) involved in somatic hyper mutation and begins the process of class switch recombination (CSR) [33]. (CSR shuffle the exons of constant region $\operatorname{IgH}$ and produces double stranded breaks but HR factor XRCC2 reduced the DSBs production by AIDs activity [33,34]. This is present in the upstream of each set of Exon's constant region of Heavy chains [32]. RAG and AID working together and exchange the segments of $\mathrm{IgH} / \mathrm{IgI}$ at high frequency in B cells [34]. The core of NHEJ have catalytic subunit (DNA-Pkcs) [35]. Ku is heterodox have two protein sub unit $\mathrm{Ku} 70$ and $\mathrm{Ku} 80$ [32,36]. $\mathrm{Ku}$ is the protein that binds to the double stranded breaks. Ku-DNA complex is important for the attachment of nuclease; polymerase and ligase of NHEJ [37-39]. Ku heterodimer play a crucial role in attachment of NHEJ factor to DSB ends. DSB are reformed by Homology direct repair (HDR) or by NHEJ. Ku acts stably and modification of DSB produces processed-ends and enhance mechanism of repair because it facilitates the NHEJ complex formation at the DSB [40]. TOPOVIB like (TOPOVIBL) is the plant and animal family of protein. TOPOVIB react and produce a complex with SP011 and involved in meiotic DSB formation [41]. RNA Helicase of the DExD/H box family cause conformational changes such as transition [42]. DNA ends come close to each other by DNAPKcs. Autophosphorylation is done by DNA-pKcs that causes conformational changes that release the DNA PKcs from DNA. NHEJ ligament complex has ligase IV (LIG4) and interact with X-ray repair cross complementing 4 (XRCC4) [43]. In Growth 1 phase of cell cycle Terminal deoxynucleotidyltransferase TDT is an enzyme identify the VDJ recombinase compounds [44].

\section{HAIR PIN FORMATION}

HA1, HA2 and HA3 are highly active protein undergoes hairpin formation on the 12-RSS when second strand is not present [45]. RAG1 produce DNA bending at the 12RSS near 60C while RAG2 not cause conformational changes [46]. pGG49 (bps 6197) is the point of breakage in DNA, their cleavage is done by RAG protein by a nick-hairpin mechanism [47]. The use of $\mathrm{Ca}^{2+}$ ion instead of $\mathrm{Mg}^{2+}$ cation changes protein stoichiometry [48]. XLF and PAXX bind to the $\mathrm{Ku}$ at the same time [32]. FHA Domain protein or fork head associated domain have proteins produced as a result of DNA damage. These proteins are related to the (PNK/PNKP) XRCC1 associated with single stranded breaks while XRCC4 associated with DSBs. PNK/PNKP Asprataxin, Aprataxin and PNK-like factor (APLF) with XRCC1 and use for repairing DNA

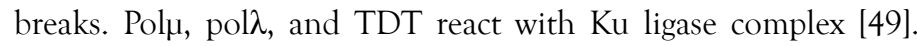
Human an T-Lymphocyte leukemia produced by SCL gene present at chromosome $1 \mathrm{p} 32 \mathrm{cRSS}$ gene also supports VDJ recombination [7]. Two zinc finger regions $(\mathrm{Zn})$ present in RAG1 are important for dimerization. $\mathrm{ZnA}$ reacts combine with RAG2 ( $\mathrm{ZnB}$ ) [9]. $\mathrm{C}_{2} \mathrm{H}_{2} \mathrm{ZnF}$ (zinc finger) domain and $\mathrm{C}$ terminal domain binds to the binary stranded DNA regulate interaction of coding sequence [11]. This region increase RSS binding and interact with RSS an collaborate with RAG1. The $\mathrm{N}$ terminal regions of RAG 1 has elements that involve in regulation of cellular protein levels, interact with zinc ions and increase cellular VDJ recombination activity. This region is known as zinc dimerization domain [11]. RAG1 and RAG2 proteins catalytic core have $384-100$ and $1-383$ residues particularly to combine. $\mathrm{ZnA}$ have ubiquit ligase activity and reacts with ubiquilates Histone H3 [50]. RAG2 non-core region bind to the histone $\mathrm{H} 3 \mathrm{~K} 4 \mathrm{Me} 3$ and start the recombination reaction [51,52]. Acidic hinge regions that take part in post cleavage mechanism, stabilisation of DNA ends and choice of repair pathway of DNA. ZnA-zinc finger domain, NBD-nanomer binding domain. 


\section{HIGH MOBILITY GROUP}

HMG1 high mobility group is the non-specific DNA binding proteins group that promotes synapsis and cleavage [9]. HMG1 and HMG2 are from the non-specific DNA binding proteins of $\mathrm{HMG}$ box family that control this activity [53,54]. HMG activity relies on metal ions. These proteins not take part in synaptic complex but start nicking and hairpin formation. Tetramer core of RSS involve in transposition [25]. Synapsis, cleavage and transposition of VDJ recombination signal's done byRAG1/2 tetramer core $[55,56]$. RAG protein binds and cuts solitary DNA strand precisely between the RSS heptamer and the coding strand of DNA. Cleavage accomplished by RAG and RSS produces four broken ends. Two 5 ' phosphorylated signal end those terminate in the RSS and 2 hairpin structures which are covalently sealed [11]. 23RSS +12 RSS come close to the RAG protein make a multi subunit synaptic complex. Signaling spread out from the interleukin-7 receptor IL7R and pre B-cell receptor control the changing model of RAG expression involved in phosphoinositide-3 kinase (P13K) and protein kinase B (also known as AKT) effect on forehead box (FOXO) transcription factor required for RAG expression [57]. DNA dependent protein kinase (DNA-PKcs) determines the hair pin structure of DNA. In non-homologous ends join of RAG DNA breaks. DNA ends have recombinant structure that gives rise to a coding joint and signal joint. NHEJ machinery has components named as PAXX modified from XRCC4 and XLF. PAXX, XRCC4 and XLF gather at the site of DNA damage to induce DSB repair and enhance DNA end ligation [43]. FOXO1 cannot bind to the enhancer region of RAG $1 / 2$ locus and give rise to the cleavage by FOXO1 [28].

\section{RECOMBINANT ANTIBODIES}

\section{Western equine encephalitis virus}

This is a solitary stranded RNA virus that is positive sense. WEE is the evolution of eastern equine encephalitis virus EEEV and Sind bis virus. This viral disease has symptoms ranging from sickness to severe encephalitis that may cause mortality. Four antibodies were originated from immune antibody gene library. The antibodies ToR68-2C3, ToR68-2E9, ToR68-3G2 and ToR69-3A2 were generated when mice were injected with a single inoculation of monoclonal antibodies. All mice were examined throughout the study, ToR68-2E9, ToR68-3G2 and ToR69-3A2 neutralized WEEV but ToR68-2C3 was not neutralized. ToR69-3A2 survival and protection was dose dependent. 200 micro gram doses gave $90 \%$ survival rate while ToR68-2C3 at high dose gave 60\% survival rate [58].

\section{Epstein bar virus}

Monoclonal neutralizing antibodies found for Epstein-Barr virus envelope glycoprotein gp350/gp220 produce in human on infection. Gp359 ectodomain has eight particular B-cell binding epitopes with CD21 and CD35. 15 new monoclonal antibodies are produced. Epstein-Barr virus glycoproteins envelop is the primary target of neutralizing antibodies. Gp350/gp220, (gp350), gp42gH are those glycoproteins that allow entry of virus into the cell, thus initiating the infection. $\mathrm{gH}, \mathrm{gL}$ band $\mathrm{gD}$ are the surface proteins encoded by virus. Gab is the type 3 transmembrane that enhances fusion of the viral and host membrane. It's activity is regulated by heterodimeric $\mathrm{gH} / \mathrm{gL}$ complex that play their role as an adapter that stimulate gab-mediated fusion on binding a cell surface receptor to target cells. B-cell infection is totally neutralized by AMMO1 that is the anti $\mathrm{gH} / \mathrm{gL}$ monoclonal antibody [59].

\section{Hepatitis}

gpE1/gpE2 give rise to cross neutralizing antibodies. HCV works on the hyper variable region. WT and HVR1 gpE1/gpE2 produce antibodies which target any type of cross-genotype neutralizing epitopes. HCV protein is immunogenic and easily tolerated in humans and animals. Chimpanzees that are vaccinated show reduced rate of $\mathrm{HCV}[60]$.

\section{Ebola virus}

GPE118, GPE325, GPE534 are targeted to different epitopes. GP antibodies show epitopes with other glycoprotein's epitopes. Resulted recombinant antibodies show high level of neutralizing activity [61].

\section{CONCLUSION}

In conclusion, DNA modifications including structural and chemical changes, some of the changes are contributing to most of the human immune system. DNA repair mechanisms are working to cause changes to allow survival, study of survival pathways are helpful to estimate the exposure and plan strategies to reduce the harmful effects of these changes on human health.

\section{ACKNOWLEDGEMENTS}

We are thankful to the institute of Molecular Biology and Biotechnology Bahauddin Zakariya university, Multan Pakistan for providing us opportunity of the publication.

\section{REFERENCES}

1. Bassing $\mathrm{CH}$, Swat W, Alt FW. The mechanism and regulation of chromosomal V (D) J recombination. Cell. 2002;109:S45-S55.

2. Schlissel M, Constantinescu A, Morrow T, Baxter M, Peng A. Double-strand signal sequence breaks in $\mathrm{V}$ (D) J recombination are blunt, 5'-phosphorylated, RAG-dependent and cell cycle regulated. Genes Dev. 1973;7:2520-2532.

3. Rosowski S, Stephen B, Lars T, Bernhard V, Simon K. A novel one-step approach for the construction of yeast surface display Fab antibody libraries. Microb Cell Fact. 2018;17:3-10.

4. Ji Y, Resch W, Corbett E, Yamane A, Schatz DG. The in vivo pattern of binding of RAG1 and RAG2 to antigen receptor loci. Cell. 2010;14:419-431.

5. Farajnia S, Ahmadzadeh V, Tanomand A, Veisi K. Development trends for generation of single-chain antibody fragments. ImmunoPharmaco and Immunotox. 2014;36:297-308.

6. Rajpal A, Strop P, Yeung YA, Pons J, Chaparro-Riggers J. Introduction: Antibody structure and function. Therap FclFusion Prot. 2014;1:44.

7. Zhang M, Swanson PC.V (D) J recombinase binding and cleavage of cryptic recombination signal sequences identified from lymphoid malignancies. J Biol Chem. 2005;283:6717-6727.

8. Bednarski JJ. RAG-mediated DNA double-strand breaks activate a cell type-specific checkpoint to inhibit pre-B cell receptor signals. J Exp Med. 2016;1:2048. 
9. Schatz DG, Swanson PC. V (D) J Recombination: Mechanisms of initiation. Annual Rev Gen. 2011;45: 167-202.

10. Helmink BA, Sleckman BP. The response to and repair of RAG-mediated DNA double-strand breaks. Ann Rev Immun. 2012;30:175-202.

11. Lescale C, Deriano L. The RAG recombinase: Beyond breaking. Mech Ageing and Develop. 2017;165:3-9.

12. De P, Zhao S, Gwyn LM, Peak MM, Rodgers KK. Thermal dependency of RAG1 self-association properties. BMC Biochem. 2008;9:1-5.

13. Ciubotaru M, Schatz DG. Synapsis of recombination signal sequences located in cis and DNA underwinding in V (D) J recombination. Mol \& Cellular Biol. 2004;24:8727-8744.

14. Swanson PC, Volkmer D, Wang L. Full-length RAG-2, and not fulllength RAG-1, specifically suppresses RAG-mediated transposition but not hybrid joint formation or disintegration. J Biol Chem. 2004;279: 4034-4044.

15. Lewis SM. Cryptic signals and the fidelity of V (D) J joining. Mol Cell Biol. 1997;17:3125-3136.

16. Drejer-Teel AH, Fugmann SD, Schatz DG. The beyond 12/23 restriction is imposed at the nicking and pairing steps of DNA cleavage during V (D) J recombination. Mol \& Cell Biol. 2007;27:6288-6299.

17. Nagawa F. Joining mutants of RAG1 and RAG2 that demonstrate impaired interactions with the coding-end DNA. J Biol Chem. 2004;279:38360-38368.

18. Wu C. Restriction of endogenous $\mathrm{T}$ cell antigen receptor $\square$ rearrangements to $\mathrm{V} \beta 14$ through selective recombination signal sequence modifications. Proceed Nat Acad Sci. 2007;104: 40024007.

19. Ru H. Molecular mechanism of V (D) J recombination from synaptic RAG1-RAG2 complex structures. Cell. 2015;163:1138-1152.

20. Kriatchko AN, Bergeron S, Swanson PC. HMG-box domain stimulation of RAG1/2 cleavage activity is metal ion dependent. BMC Mole Biol. 2008;9:32.

21. Ciubotaru M, Kriatchko AN. Fluorescence resonance energy transfer analysis of recombination signal sequence configuration in the RAG1/2 synaptic complex. Mol \& Cell Biol. 2007;27:4745-4758.

22. Shlyakhtenko LS. Molecular mechanism underlying RAG1/RAG2 synaptic complex formation. J Biol Chem. 2009;284:20956-20965.

23. Swanson PC. A RAG-1/RAG-2 tetramer supports 12/23-regulated synapsis, cleavage and transposition of $\mathrm{V}(\mathrm{D}) \mathrm{J}$ recombination signals. Mol \& Cell Bio. 2002;22:7790-7801.

24. Grundy GJ, Hesse JE, Gellert M. Requirements for DNA hairpin formation by RAG1/2. Proceed Nat Acad Sci. 2007;104:3078-3083.

25. Bergeron S, Anderson DK, Swanson PC. RAG and HMGB1 proteins: purification and biochemical analysis of recombination signal complexes. Methods Enzymol. 2006;408:511-528.

26. Lieber MR. The mechanism of double-strand DNA break repair by the nonhomologous DNA end-joining pathway. Ann Rev Bio Chem. 2010;79:181-211.

27. Chatterjee N, Walker GC. Mechanisms of DNA damage, repair and mutagenesis. Environ Mol Mutag. 2017;58:235-263.

28. Ochodnicka-Mackovicova K. The DNA Damage Response Regulates RAG1/2 Expression in Pre-B Cells through ATM-FOXO1 Signaling. J Immun. 2016;197:2918-2929.

29. Arya R, Bassing CH. V (D) J recombination exploits DNA damage responses to promote immunity. Trend Gen. 2017;33:479-489.

30. Thornton TM. Inactivation of nuclear GSK3 $\beta$ by Ser 389 phosphorylation promotes lymphocyte fitness during DNA doublestrand break response. Nat Comm. 2016;7:10553.

31. Fisher MR. Immature lymphocytes inhibit Rag1 and Rag2 Transcription and V (D) J recombination in response to DNA double-strand breaks. J Immun. 2017;198: 2943:2956.

32. Kumar V, Alt FW, Oksenych V. Functional overlaps between XLF and the ATM-dependent DNA double strand break response. DNA Rep. 2014;16:11-22.

33. Hasham MG. Widespread genomic breaks generated by activationinduced cytidine deaminase are prevented by homologous recombination. Nat Immun. 2010;11:820.

34. Wang JH. Mechanisms promoting translocations in editing and switching peripheral B cells. Nature. 2009; 460:231-236.

35. Morales AJ. A type I IFN-dependent DNA damage response regulates the genetic program and inflammasome activation in macrophages. Elife. 2017;6 e24655.

36. Rulten SL, Grundy GJ. Non-homologous end joining: common interaction sites and exchange of multiple factors in the DNA repair process. Bioessays. 2017;39:1600209.

37. Wang JH. Oncogenic transformation in the absence of Xrcc4 targets peripheral B cells that have undergone editing and switching. J Exper Med. 2008;205:3079-3090.

38. Amzel LM. Structural comparisons of class I phosphoinositide 3-kinases. Nat Rev Cancer. 2008;8:665-669.

39. Walker EH. Structural determinants of phosphoinositide 3-kinase inhibition by wortmannin, LY294002, quercetin, myricetin and staurosporine. Mol Cell. 2000;6: 909-919.

40. Emerson $\mathrm{CH}$. Ku DNA End-Binding Activity Promotes Repair Fidelity and Influences End-Processing During Nonhomologous End-Joining in Saccharomyces cerevisiae. Genetics. 2018;209:115-128.

41. Robert T, Nore A, Brun C, Maffre C, Crimi B. The TopoVIB-Like protein family is required for meiotic DNA double-strand break formation. Sci. 2016;351:943-949.

42. Papasaikas P, Valcárcel J. The Spliceosome: The ultimate RNA chaperone and sculptor. Trends Biochem Sci. 2016;4:33-45.

43. Hammel M. An intrinsically disordered APLF links Ku, DNA-PKcs and XRCC4-DNA ligase IV in an extended flexible non-homologous end joining complex. J Biol Chem. 2016;291:26987-27006.

44. Tauraite D. Modified nucleotides as substrates of terminal deoxynucleotidyl transferase. Mol. 2017;22: 672.

45. Nishihara T. RAG-heptamer interaction in the synaptic complex is a crucial biochemical checkpoint for the $12 / 23$ recombination rule. J Biol Chem. 2008;283:4877-4885.

46. Pavlicek JW, Lyubchenko YL, Chang Y. Quantitative Analyses of RAG-RSS Interactions and Conformations Revealed by Atomic Force Microscopy. Bio Chem. 2008;47:11204-11211.

47. Zhang M, Swanson PC. HMGB1/2 can target DNA for illegitimate cleavage by the RAG1/2 complex. BMC Mole Biol. 2009;10: 24 .

48. Nagawa F, Kodama M, Nishihara T, Sakano H. Footprint analysis of recombination signal sequences in the $12 / 23$ synaptic complex of $\mathrm{V}$ (D) J recombination. Mole \& Cell Biol. 2002;22:7217-7225.

49. Cherry AL. Versatility in phospho-dependent molecular recognition of the XRCC1 and XRCC4 DNA-damage scaffolds by aprataxinfamily FHA domains. DNA Repair. 2015;35:116-125.

50. Bettridge J. H3K4me3 induces allosteric conformational changes in the DNA-binding and catalytic regions of the $\mathrm{V}(\mathrm{D}) \mathrm{J}$ recombinase. Proceedings of the National Academy of Sciences. 2017;114:1904-1909. 
51. Deng Z, Liu H, Liu X. RAG1-mediated ubiquitylation of histone $\mathrm{H} 3$ is required for chromosomal V (D) J recombination. Cell Res. 2015;25:181.

52. Maman Y, Teng G, Seth R, Kleinstein SH, Schatz GG. RAG1 targeting in the genome is dominated by chromatin interactions mediated by the non-core regions of RAG1 and RAG2. Nucleic Acid Res. 2016; 44:9624-9637.

53. Keeney S, Giroux CN, Kleckner N. Meiosis-specific DNA doublestrand breaks are catalyzed by Spol1 a member of a widely conserved protein family. Cell. 1997;88:375-384.

54. Bergeron S, Madathiparambil T, Swanson PC. Both high mobility group (HMG)-boxes and the acidic tail of HMGB1 regulate recombination-activating gene (RAG)-mediated recombination signal synapsis and cleavage in vitro. J Biolo Chem. 2005;280:31314-31324.

55. Swanson PC. A RAG-1/RAG-2 Tetramer Supports 12/23-Regulated Synapsis, Cleavage and Transposition of V (D) J Recombination Signals. Mol Cell Biol. 2003;23:3029.
56. Zagelbaum J. Real-time analysis of RAG complex activity in V (D) J recombination. Proceed Nat Acad Sci. 2016;113:11853-11858.

57. Ochodnicka-Mackovicova K. NF-kB and AKT signaling prevent DNA damage in transformed pre-B cells by suppressing RAG1/2 expression and activity. Blood. 2015;126:1324-1335.

58. Burke CW. Human-Like Neutralizing Antibodies Protect Mice from Aerosol Exposure with Western Equine Encephalitis Virus. Viruses. 2018;10:147.

59. Mutsvunguma LZ. Identification of a Novel Neutralizing and Two Non-Neutralizing Epitopes on Epstein-Barr Virus gp350 Protein. Bio Rxiv. 2018;30:2844.

60. Sherman KE, Mitty J, Saab S, Chak E, Talal AH. Hepatitis E virus infection in USA: an estimate of true prevalence 2010;31:1090-1101.

61. Panina AA, Dementieva IG, Aliev TK, Toporova VA, Balabashin DS. Recombinant Antibodies to the Ebola Virus Glycoprotein. Acta Naturae. 2017;9: 84-91. 